\title{
Effect of Object Frequency on Focal Position of Four Photographic Objectives
}

\author{
Fred W. Rosberry
}

\begin{abstract}
Three wide-angle aerial-camera lenses of 6-inch nominal focal length and one smaller lens of 32-millimeter focal length were given photographic resolving-power tests. Targets were of the parallel line type with several groups of three lines representing a wide range of lines-per-millimeter values. The image plane of best focus was visually located, and exposures were made at four different positions inside of this focus and the same number outside. Measurement of the resultant negatives by a recording microdensitometer revealed the image contrast of the various line-space frequency groups at the nine focal positions. Curves are presented showing the focal positions of maximum image contrast for four values of line-space frequency.
\end{abstract}

\section{Introduction}

During the past decade, there has been considerable interest in the effect of target contrast on image contrast and resolving power in the image plane. Investigation of the relationships between these quantities has shown the existence of a number of associated phenomena, among which is a shift in the plane of best focus with target pattern frequency. Any appreciable shift will affect the selection of the proper focal plane in a fixed-focus camera, such as an aerial camera $[1,2] .^{1}$ The Optical Instruments laboratory has therefore made an investigation of this phenomenon.

Of the lenses on hand at the time this investigation was being planned, three, representing a good, medium, and fair quality as shown by previous resolving power tests, were chosen for testing. They were all wide-angle aerial camera lenses of nominally 6 -in. focal length, made by the same manufacturer. A fourth lens was later included representing a different type and of only $32-\mathrm{mm}$ (1.3-in.) focal length.

\section{Apparatus and Procedure}

To measure the contrast rendition, or contrast in the image plane of a lens, by a photographic procedure, extreme care had to be taken to control the other factors that can affect contrast. Some of these factors are exposure, development, and variation in emulsion from one photographic plate to another. Uniform exposure was maintained by the use of an automatic timing device, with relatively long exposure times such as 2 or 3 sec instead of small fractions of a second as obtained by shutters. Development was maintained uniform by putting as many different exposures as possible on one plate and by agitation during development. The variation in characteristics between plates out of the same box is very small, but this was further reduced by cutting large plates into smaller ones, thus making two or more from one.

Figures in brackets indicate the literature references at the end of this paper.
The targets used in this investigation were the 75-line step contrast and the 200-line step contrast targets, which are variations of those previously reported [3]. The 75-line target consists of 25 groups of 3-line patterns. The line widths and spacings in a given pattern are equal; however, the values vary from one pattern to the next by a ratio equal to $\sqrt[6]{2}$. The range of resolving power for this master target is therefore from 0.2 to 3.2 lines $/ \mathrm{mm}$. The range of the 200-line target is from 0.2 to 2 lines $/ \mathrm{mm}$, which means that the actual widths of lines and spaces vary from 2.5 to $0.25 \mathrm{~mm}$. This range is covered in a continuously varying linear manner with 200 lines and 200 spaces contained in a distance of $180 \mathrm{~mm}$. The zero line is $20 \mathrm{~mm}$ from the origin, so that the over-all length of the resolving-power portion of the chart is $200 \mathrm{~mm}$ (fig. 1). The length of the lines is $185 \mathrm{~mm}$, thus giving the contrast scale approximately the same length as the frequency scale. Along the length of the lines the targets are divided into nine areas of different line-space contrast values. The 75-line target had a contrast range in these nine areas from 0.22 to a high of 3.19 on the density scale. The same nine areas on the 200-line target had a range from 0.10 to 1.51 .

Although two targets were used in this investigation, they were not used simultaneously. The target under observation was mounted on the axis and in front of the lens being tested. As the targets are transparencies, it was necessary for them to be uniformly illuminated from behind. This was accomplished by using a light box of such design that only diffusely reflected light, which was quite uniform and free of hot spots, fell upon the target.

The camera used for exposing the test plates consists of two major components, namely, the lens mount and the camera back (fig. 2). A set of ways similar to a lathe bed supports both components, each mounted on its own carrier or saddle, thus allowing for the necessary movement of one with respect to the other by means of a lead screw. This movement is necessary to accommodate lenses of different focal lengths. The camera back itself is provided with three micrometer screws, by means 


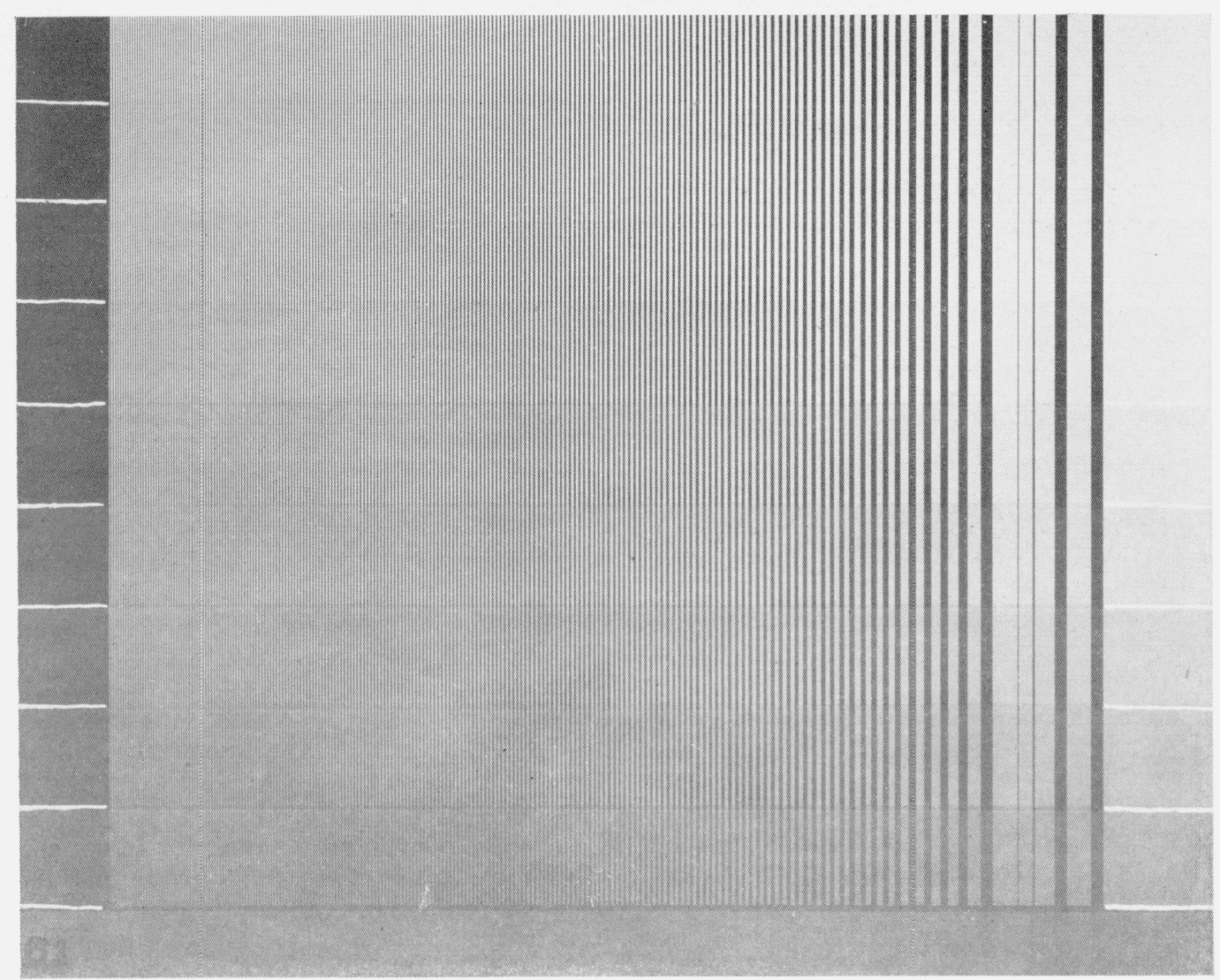

FIGURE 1. The 200-line step contrast target as used in this investigation The 75-line target was similar except for the 3-line grouping of the lines in the pattern.

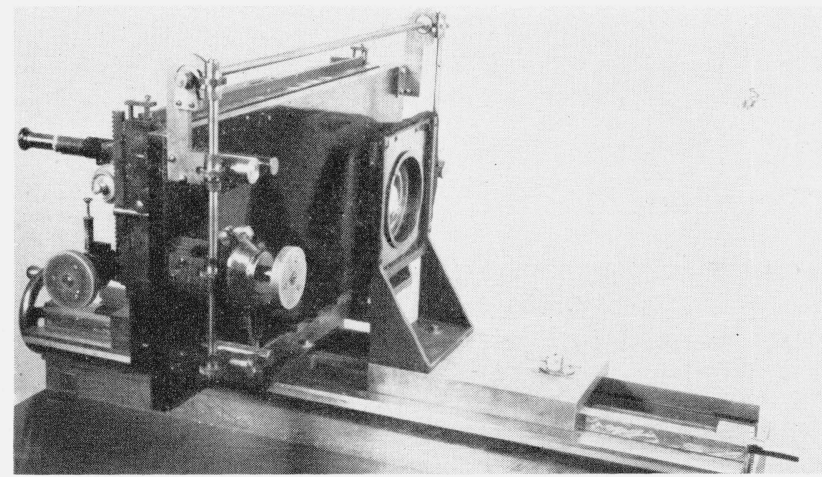

Figure 2. Bench showing lens, plate carrier, and focusing microscope. 
of which small precisely measured axial displacements of a millimeter or less may be made. The plate holder can also be moved vertically in a plane perpendicular to the lens axis, allowing successive images to be taken on a single plate, one above the other. Changes in the focal position of amounts as small as $0.2 \mathrm{~mm}$ are easily accomplished with the micrometer adjustment. A microscope is mounted on the same saddle as the camera back and focused on the plane of the emulsion.

The lens mount supports the lens rigidly in place and carries the necessary bellows and light shields to protect the negative from stray light. The mount is constructed so that it holds a metal lens board measuring 6 in. square, which in turn carries the lens under test.

The distance between lens and target was equal to 26 focal lengths of the lens under test. With this lens-to-target spacing the image distance is a little longer than for an ininitely distant target, but it is believed that there is very little change in the resolving power characteristics. Theoretical considerations indicate that the lens-to-target distance can be reduced to as low as 15 focal lengths without significant change in the resolution characteristics from those for infinitely distant objects. ${ }^{2}$

With the lens-target distance set at 26 focal lengths for the 6-in. lenses, the effective spacing of lines in the target on the image plane was 5.0 to 79.6 lines/ $\mathrm{mm}$ for the 75 -line target. With the $32-\mathrm{mm}$ lens, the lens-to-target distance was 51 focal lengths, giving a range of 10 to 100 lines $/ \mathrm{mm}$ for the 200 -line target. Focusing was accomplisbed by properly spacing the camera-back and the lens until a well defined image appeared on a ground glass, which was in the position the photographic plate would occupy during photographing. The microscope was then focused on the plane of the ground glass and locked in that position. With the ground glass removed, it was then possible to view the image directly and make any small focusing adjustments necessary. The micrometer was then set so that the plate carrier was $0.8 \mathrm{~mm}$ inside of the best visual focus, and a panchromatic photographic plate set in the carrier. Using the vertical ways of the camera back, the plate was racked to its uppermost position and the first exposure made. The plate was then moved away from the lens by $0.2 \mathrm{~mm}$ and lowered enough to allow the image to be put above the first and another exposure made. This procedure was repeated until nine exposures were made, covering the range from $0.8 \mathrm{~mm}$ inside to $0.8 \mathrm{~mm}$ outside of the visually estimated focus. After developing in Dk-60a for 5 min and fixing, this plate was then ready for measurement.

The density of each line and space for three of the target contrast levels were the quantities to be measured. These were determined by using a recording microdensitometer. The test negative was

${ }^{2}$ Private communication from D. P. Feder. located on the plate carrier of the instrument, properly oriented, and brought into good focus. One contrast area of one focal position of the test negative was then scanned and the local values of line and space density were automatically recorded in the form of a graph. The negative was then relocated so that the same contrast area on the next adjacent image, representing the next focal position, was then ready for scanning. The instrument was refocused at each change in negative position. All focal positions were covered by this procedure, and this constituted a run. Other runs were made for the two remaining contrast areas to be measured. Each run was repeated two more times with the results averaged to minimize any variation in measurement that still might exist, thus making three runs of the three contrast areas, with each run covering the nine focal positions.

\section{Results of Measurement}

The graph produced by the microdensitometer consisted of curves on a strip of chart paper graduated with a logarithmic scale of photographic density whose limits were zero and infinity. The quantity measured was the amount of light transmitted by a very small area of the test negative. Because of the line and space characteristic of the negative being scanned, the recording pen was traveling from near one end of the scale to near the other, thus indicating the highest transmission or minimum density at a space and the maximum density at a line. These data were tabulated by reading, on the $\log$ scale, the maximum and minimum for each cycle. The difference of the two was called contrast (C). The 75 -line target, which was divided into 25 groups of 3 lines and had the line frequency constant for each group, was averaged to determine the maximums and minimums for each group. A graphical representation of these data is shown in figures 3 and 4 . The curves are grouped according to the particular lens under investigation and the target contrast. Each curve represents the amount of contrast in the image at various focal positions for a speciifed image frequency. Some of the curves show a bar indicating the calculated depth of focus for the adjacent frequency or resolving power. The depths of focus herein referred to were calculated from the formula $d=4 b / a$, where $d$ is the depth of focus in millimeters, $b$ is the $f$-number, and $a$ is the resolving power in lines per millimeter, for which the depth of focus is sought [3]. This formula was developed for use with high-contrast targets, which should be taken into account when viewing the results. Theoretically the curve should reach zero value of image contrast at about the same points as indicated by the ends of the depth-of-focus line. The range of the depth of focus is shown only for the two curves of highest frequency, as the range of focal positions is not great enough to include the depth-of-focus range at the lower frequencies. 

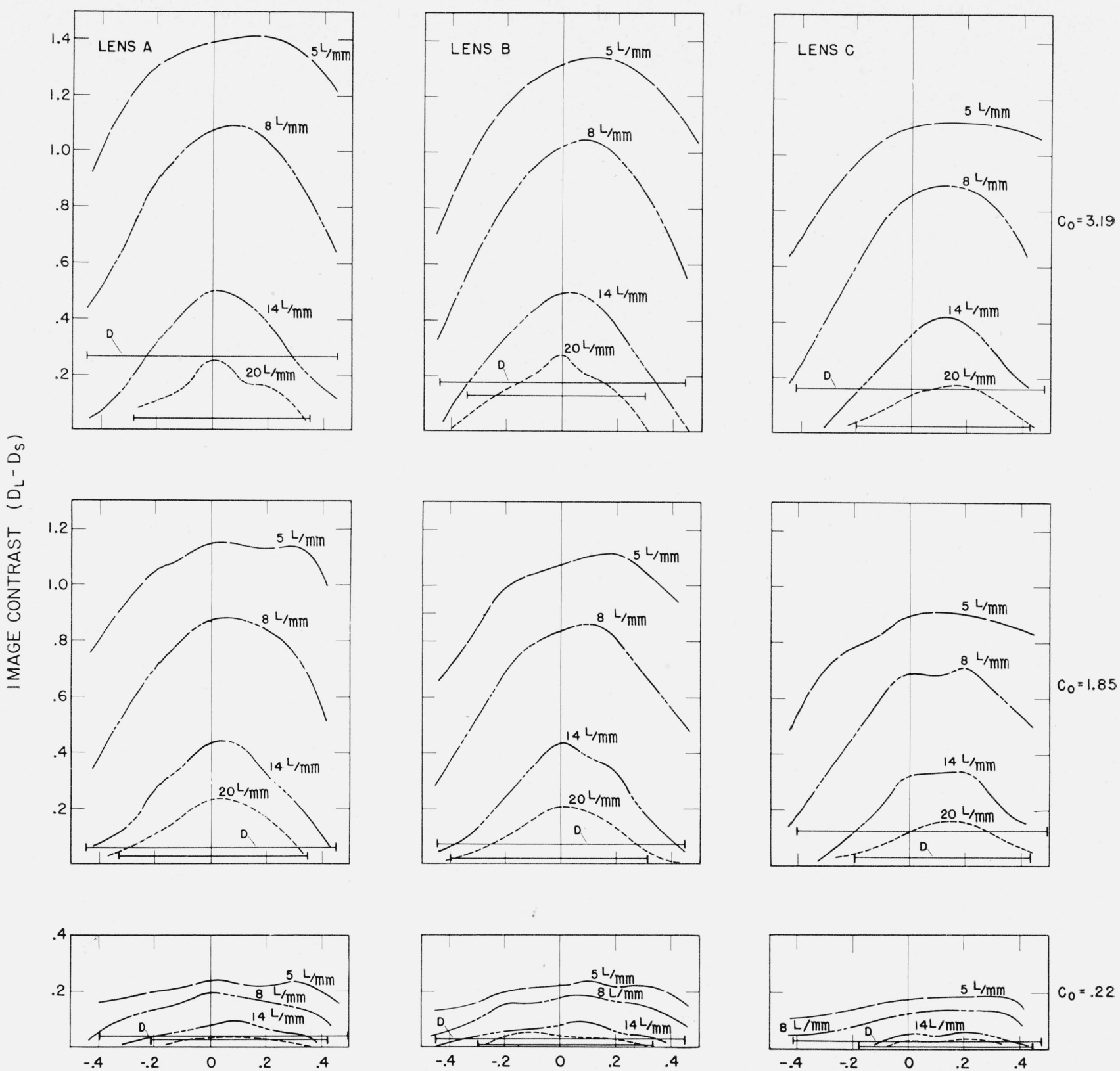

FOCAL POSITION, Mm

Figure 3. Curves showing the contrast in the image at various focal positions of four line-frequency patterns at three values of target contrast for the three wide-angle aerial camera lenses.

$L / \mathrm{mm}$, lines per millimeter; $D_{\mathrm{L}}$, photographic density of line; $D_{\mathrm{s}}$, photographic density of space. The lines marked " $D$ " indicate the range of the calculated depth of focus. 

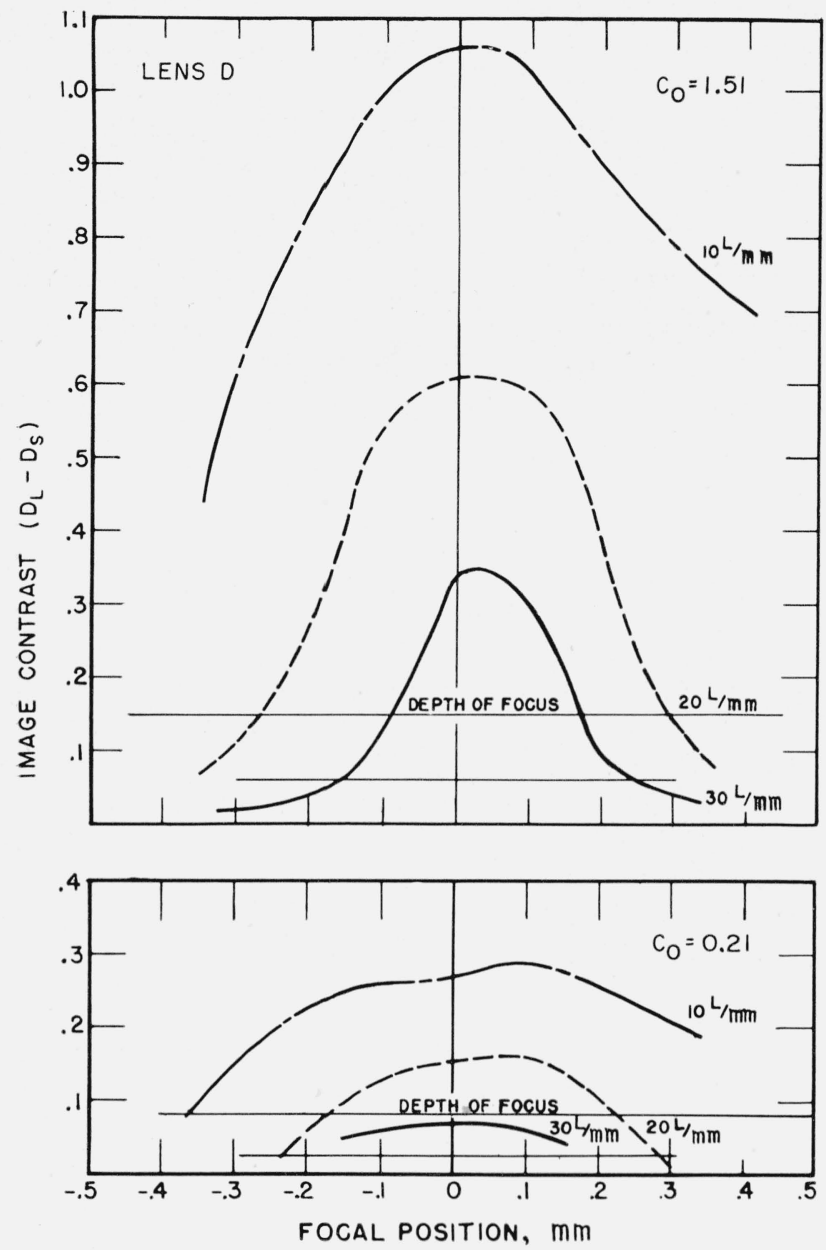

Figure 4. Contrast-focal position curves for $32-m m$ lenses. These curves are similar to those of figure 3 for 6 -in. lenses.

\section{Discussion}

Contrast can be expressed as the difference between two densities $(\Delta D)$, or it can be expressed as a ratio of the two transmissions $T_{1} / T_{2}$. Botb methods are in use, with perhaps the former more common. Some of the curves were plotted by both methods, and although each illustrated the point well, the $\Delta D$ procedure lent itself better to data from the microdensitometer. One point rather clearly shown by these curves is the relatively small amount of shift in the optimum focus for the four frequencies plotted. This of course is a quality that will change from one lens to another and is somewhat dependent on the individual lens aberrations.

The three wide-angle aerial camera lenses were given resolution tests prior to this investigation, and the results of these tests established that their resolution characteristics were within the range covered by the average lens of this type. Distortion measurements also indicate that the sample lenses are not outside the average. The lenses tested herein are

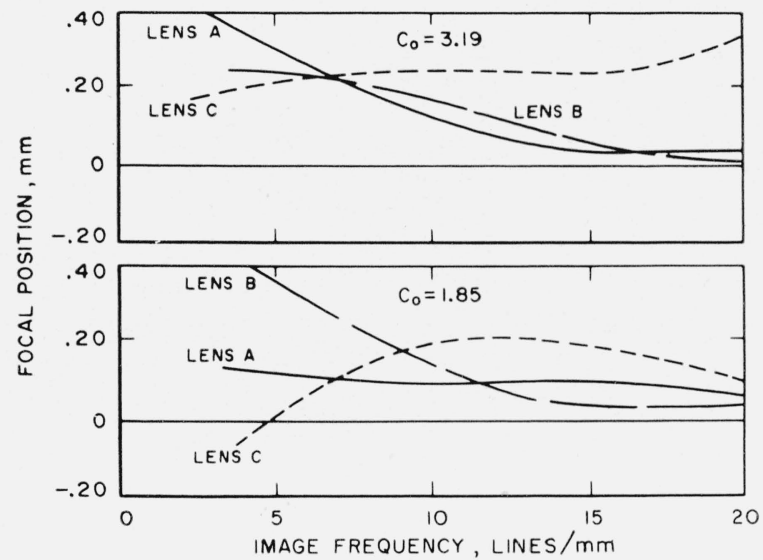

FIGURE 5. Curves showing the shift from an arbitrary zero of the focal position giving highest contrast for various line frequencies at two target-contrast levels.

The same three lenses are shown in figure 4.

quite consistent in showing very little shift of focus at the two higher target contrasts; at the low target or object contrast $\left(\vec{C}_{0}=0.22\right)$ the shift is even less noticeable. The sets of curves shown in figure 5 summarize the findings for the three 6 -in. wideangle lenses. The amount of shift (from an arbitrary zero) of the focal position giving highest contrast is plotted for four values of image frequency. The two plots represent the results obtained with two different target contrasts. The focal position measured along the ordinate of the graph was determined from the crest of the corresponding contrast curve shown in figure 3. The contrast curves for the lower frequencies are rather flat-topped, which makes precise location of the peaks difficult. Shifting of the plane of best focus with object frequency as indicated herein is very small for lenses of near average quality.

As focal settings become more precise, the position of best focus becomes more difficult to locate. A zone is found within which a position of best resolution and another position of highest contrast rendition are located. This effect is present at all points in the field and, as the range of this zone of best focus is less than the inherent field curvature of a lens, critical focusing over the entire field becomes extremely difficult. The residual spherical aberration in the lens is probably a cause of this focal shift. The position for maximum contrast of broad lines is where the circle of confusion is the smallest and not necessarily where the resolution is best.

The present work accordingly confirms the results of earlier workers $[1,2,5]$ that a difference in focal setting exists between the settings for best resolution and for best contrast rendition. However, it emphasizes the fact that this difference is usually so small that it is seldom advantageous to set for the position of maximum contrast rendition for lowfrequency patterns as opposed to setting for the position of maximum resolution. For example, in some earlier work $[1,2]$, the difference in focal setting 
for frequencies in the image of 4.1 and 20.6 lines $/ \mathrm{mm}$ was indicated to be as high as 0.03 in., or $0.76 \mathrm{~mm}$. It was further indicated that the improvement in contrast rendition would be quite appreciabie at the lower frequencies in setting at the optimum image contrast for those frequencies with marked loss at the higher frequencies. It is clear from the results shown in this paper for the three lenses, shown in figure 3 , that the shift in focal settings between frequencies of 5 and 20 lines $/ \mathrm{mm}$ is at most $0.20 \mathrm{~mm}$. Moreover, the slight gain in contrast rendition by setting at the maximum for the low frequency is not sufficient to warrant accepting the appreciable loss in contrast rendition at the higher frequencies. It is probable that some few lenses of a given type show focal shifts as high as $0.76 \mathrm{~mm}$, but it seems likely that such lenses are the exception rather than the rule.
The author expresses appreciation for the interest and advice rendered by Francis E. Washer and Robert E. Stephens.

\section{References}

[1] Duncan E. MacDonald, Photogrammetric Eng. 17, 383 (1951).

[2] Robert N. Colwell, Photogrammetric Eng. 20, 433 (1954).

[3] Francis E. Washer and Fred W. Rosberry, J. Opt. Soc. Am. 41, 597 (1951).

[4] Francis E. Washer, J. Research NBS 34, 187 (1945) RP1636.

5] R. N. Hotchkiss, F. E. Washer, and F. W. Rosberry, J. Opt. Soc. Am. 41, 600 (1951).

Washington, November 20, 1955. 